\title{
Unusual context of CENPJ variants and primary microcephaly: compound heterozygosity and nonconsanguinity in an Argentinian patient
}

\author{
Anna M. Cueto-González (10) ${ }^{1,2,3}$, Mónica Fernández-Cancio ${ }^{4,5}$, Paula Fernández-Alvarez ${ }^{1,2}$, Elena García-Arumí1,5,6 and
} Eduardo F. Tizzano 1,2,3,5

\begin{abstract}
Primary microcephaly $(\mathrm{MCPH})$ is a genetically heterogeneous disorder showing an autosomal recessive mode of inheritance. Patients with MCPH present head circumference values two or three standard deviations (SDs) significantly below the mean for age- and sex-matched populations. MCPH is associated with a nonprogressive mild to severe intellectual disability, with normal brain structure in most patients, or with a small brain and gyri without visceral malformations. We present the case of an adult patient born from Argentinian nonconsanguineous healthy parents. He had a head circumference $>5$ SD below the mean, cerebral neuroimaging showing hypoplasia of the corpus callosum, bilateral migration disorder with heterotopia of the sylvian fissure and colpocephaly. The patient was compound heterozygous for pathogenic variants in the CENPJ gene (c.289dupA inherited from his mother and c.1132 C > T inherited from his father). Our patient represents an uncommon situation for the usual known context of CENPJ and MCPH, including family origin (Argentinian), pedigree (nonconsanguineous), and genotype (a compound heterozygous case with two variants predicting a truncated protein). Next-generation sequencing studies applied in a broader spectrum of clinical presentations of $\mathrm{MCPH}$ syndromes may discover additional similar patients and families.
\end{abstract}

Primary microcephaly $(\mathrm{MCPH})$ is a genetically heterogeneous congenital disorder of brain development. Most cases of MCPH show an autosomal recessive mode of inheritance. Affected patients present head circumference values equal to or greater than two to three standard deviations (SDs) below the age- and sex-matched population mean. $\mathrm{MCPH}$ is associated with a nonprogressive mild to severe intellectual disability and the absence of visceral malformations ${ }^{1,2}$. There are some discrepancies with the parameters to define and consider $\mathrm{MCPH}$ in a patient. A proportion of papers define $\mathrm{MCPH}$ as having a head circumference value $\geq 2$ SDs below the population

\footnotetext{
Correspondence: Anna M. Cueto-González (amcueto@vhebron.net)

'Department of Clinical and Molecular Genetics, Vall d'Hebron Barcelona

Hospital Campus, Barcelona, Spain

${ }^{2}$ Medicine Genetics Group, Vall d'Hebron Research Institute (VHIR), Vall

d'Hebron Barcelona Hospital Campus, Autonomous University of Barcelona Barcelona, Spain

Full list of author information is available at the end of the article
}

mean $(\sim 2 \%$ of the general population is included in this definition), and others consider a value that is $\geq 3$ SDs below the mean $(0.1 \% \text { of the population is included })^{1}$. $\mathrm{MCPH}$ is caused by mutations in at least 25 different genes involved in DNA damage repair, orienting the mitotic spindle, or in cell division control ${ }^{3-6}$ (Table 1).

We present the case of an adult patient born in Argentina from Argentinian nonconsanguineous parents. Next-generation sequencing (NGS) of MCPH genes revealed a compound heterozygous genotype with two pathogenic variants in CENPJ (OMIM 609279).

Our patient is, at present, 20-years old, and he has been followed-up in several Argentinian and Spanish hospitals because of his clinical manifestations. He is the only son of an Argentinian healthy nonconsanguineous couple (age of parents at birth, mother 19-years old and father 26years old). No other relevant familial pathological cases were reported. His mother had only one echography at 
Table 1 Update of the 25 subtypes of primary autosomal recessive microcephaly and related causative genes; $\mathrm{MCPH}=$ primary microcephaly (compiled from ref. ${ }^{5}$ ).

\begin{tabular}{|c|c|c|c|}
\hline $\begin{array}{l}\text { MCPH } \\
\text { subtype }\end{array}$ & $\begin{array}{l}\text { OMIM } \\
\text { phenotype number }\end{array}$ & Gene & $\begin{array}{l}\text { OMIM } \\
\text { genotype number }\end{array}$ \\
\hline $\mathrm{MCPH} 1$ & 251200 & $\mathrm{MCPH} 1$ & 607117 \\
\hline $\mathrm{MCPH} 2$ & 604317 & WDR62 & 613583 \\
\hline MCPH3 & 604804 & CDK5RAP2 & 608201 \\
\hline $\mathrm{MCPH} 4$ & 604321 & KNL1 & 609173 \\
\hline MCPH5 & 608716 & ASPM & 605481 \\
\hline MCPH6 & 608393 & CENPJ & 609279 \\
\hline $\mathrm{MCPH7}$ & 612703 & STIL & 181590 \\
\hline MCPH8 & 614673 & CEP135 & 611423 \\
\hline MCPH9 & 614852 & CEP152 & 613529 \\
\hline MCPH10 & 615095 & ZNF335 & 610827 \\
\hline MCPH11 & 615414 & PHCl & 602978 \\
\hline MCPH12 & 616080 & CDK6 & 603368 \\
\hline MCPH13 & 616051 & CENPE & 117143 \\
\hline MCPH14 & 616402 & SASS6 & 609321 \\
\hline MCPH15 & 616486 & MFSD2A & 614397 \\
\hline MCPH16 & 616681 & ANKLE2 & 616062 \\
\hline MCPH17 & 617090 & CIT & 605629 \\
\hline MCPH18 & 617520 & WDFY3 & 617485 \\
\hline МСPH19 & 617800 & COPB2 & 606990 \\
\hline MCPH2O & 617914 & KIF14 & 611279 \\
\hline MCPH21 & 617983 & NCAPD2 & 615638 \\
\hline MCPH22 & 617984 & NCAPD3 & 609276 \\
\hline MCPH23 & 617985 & NCAPH & 602332 \\
\hline MCPH24 & 618179 & NUP37 & 609264 \\
\hline MCPH25 & 618351 & MAP11 & 618350 \\
\hline
\end{tabular}

3 months of pregnancy, and normal results were reported. No environmental factors, such as infections or drugs, were noted during pregnancy. The patient's birth weight was $2.8 \mathrm{~kg}(-1.38 \mathrm{SD})$, birth length was $48 \mathrm{~cm}(-1.44 \mathrm{SD})$, and head circumference was $30.5 \mathrm{~cm}(-3.05$ SD). Psychomotor delay began during infancy, and progressively, moderate to severe intellectual disability was noted, together with hyperactivity. The present physical examination shows a head circumference of $48 \mathrm{~cm}(-5.42 \mathrm{SD})$ with height and weight in the third percentile. His height $(167 \mathrm{~cm},-1.63 \mathrm{SD})$ is lower than his mean parental height $(179 \pm 5 \mathrm{~cm},+0.24 \mathrm{SD}$; mother is $170 \mathrm{~cm}$ and father is $175 \mathrm{~cm}$ ). Severe $\mathrm{MCPH}$, a sloping forehead, a long nasal columella, markedly crowded teeth, and mild pectus excavatum were present. No other relevant phenotypic characteristics were noted. The parents (legal guardians) gave written informed consent (approved by Vall $\mathrm{d}$ 'Hebron Ethical Committee) for biochemical and molecular studies, but refused to permit pictures to be taken of the patient. A number of complementary tests were performed, including karyotype $(46, \mathrm{XY})$ and comparative genome hybridization array (reported as normal); metabolic screening (normal); the exclusion of maternal phenylketonuria; echocardiogram (normal); and cerebral magnetic resonance imaging (performed at 9 years, reporting hypoplasia of the corpus callosum, bilateral migration disorder with heterotopia of the sylvian fissure and colpocephaly).

Genomic DNA of the patient and his parents was extracted from peripheral blood mononuclear cells using standard procedures. For library preparation, a commercially available targeted resequencing kit, the TruSight One (TSO) Sequencing Panel, was used, and the DNA samples were sequenced on a MiSeq platform (Illumina, San Diego, CA, USA). The TSO Sequencing Panel kit allows the analysis of 4813 target genes associated with human genetic disorders, including 38 autosomal recessive $\mathrm{MCPH}$ genes related to isolated $\mathrm{MCPH}$, and other recessive entities with primary $\mathrm{MCPH}$ and other malformations (example: the TUBGCP6 and PLK4 genes, which are related to $\mathrm{MCPH}$ associated with chorioretinopathy; http://www.illumina.com/products/trusightone-sequencing-panel.html). All procedures were prepared according to the manufacturers' instructions. Data were analyzed using MiSeq Reporter software (Illumina) and mapped to the human genome reference sequence (GRCh37, hg19).

For data analysis, we used VariantStudio version 2.2.1 (Illumina) and Integrative Genomics Viewer software version 2.3.57 (Broad Institute, Cambridge, MA, USA). The DNA sample of this patient generated $\sim 9700$ variants. Filtering criteria included a coverage of $>20$ reads, a variant frequency of $>20 \%$, and a minor allele frequency of $<0.02 \%$ in the 1000 Genomes database and the gnomAD browser (http://gnomad.broadinstitute.org; NHLBI Exome Sequencing Project), and selected for variant types, such as missense, nonsense, synonymous, and splice variants. The first analysis filtered for a recessive mode of inheritance in candidate genes for autosomal recessive $\mathrm{MCPH}$. The pathogenicity of variants was ascertained according to the criteria of the American College of Medical Genetics ${ }^{7}$.

Two pathogenic variants were detected: c.289dupA (p. (Thr97AsnfsTer7); reported by our group in ClinVar: SCV000986655) and c.1132 C > T (p.(Arg378Ter)) in the CENPJ gene (NM_018451.4, NP_060921.3; reported by our group in ClinVar: SCV000986656). These variants were confirmed by Sanger sequencing (Fig. 1). His mother is a c.289dupA carrier, and his father is a c. $1132 \mathrm{C}>\mathrm{T}$ carrier. 
c.289dupA/p.Thr97AsnfsTer7

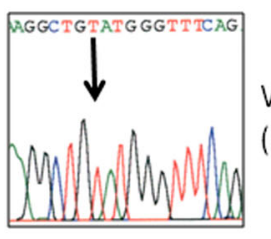

Wild-type

(reverse sequence)

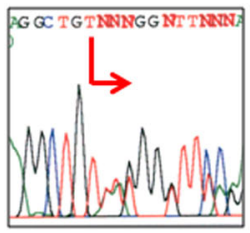

Maternally

inherited

variant

(reverse

sequence)
c.1132C >T/p.Arg378Ter
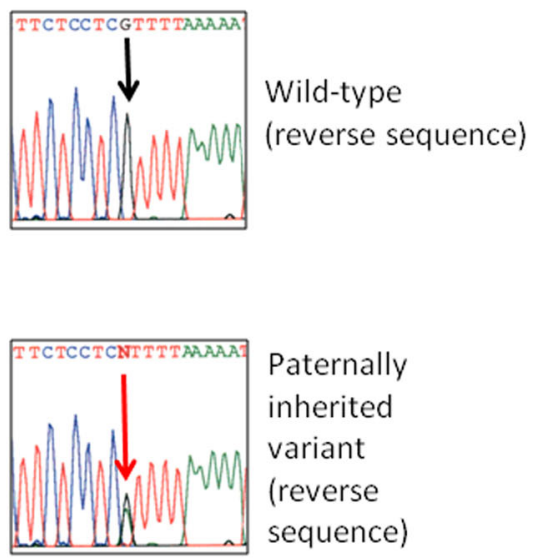

Paternally

inherited

variant

(reverse

sequence)

Fig. 1 Electropherogram of the two pathogenic variants found in our patient. The upper part shows the wild-type genotype, and the lower part shows the genotype of the patient (reverse sequences).

Both mutations were found in the gnomAD browser with frequencies of $0.014 \%$ and $0.012 \%$, respectively, in the Latino population. The two variants were considered pathogenic because both predicted the generation of a truncated protein of 103 and 378 amino acids, respectively, instead of the 1338 amino acids of the wild-type protein. Both variants were considered deleterious by in silico predictors (UMD predictor, PROVEAN, Mutation Taster).

This is the first report of an MCPH patient who is compound heterozygous for two truncating variants in CENPJ. To the best of our knowledge, this is also the first case of Argentinian origin reported, given that these patients are usually more common in other populations with higher inbreeding rates. To date, all the mutations identified in the CENPJ gene have been reported in a homozygous state, and the vast majority have been reported in consanguineous couples from Pakistan, Brazil, southern Saudi Arabia, and Turkey (Table 2 compiles families and variants reported so far, including our patient). All these reported variants were novel, with the exception of c.18delC (p.Ser7Profs*2), which was reported to be recurrent in Pakistani patients ${ }^{8}$. CENPJ pathogenic variants have been described in patients with autosomal recessive primary MCPH type 6 (OMIM 608393) ${ }^{9,10}$, Seckel syndrome type 4 (OMIM 613676) ${ }^{11} /$ primordial dwarfism, and in a patient with arthrogryposis and abnormal scar formation, although this last patient had normal growth parameters, including head circumference $^{6,12}$ (Table 2). Primary MCPH and primordial dwarfism are considered to belong to a continuous spectrum of phenotypic manifestations, without a clear genotype-phenotype correlation ${ }^{6}$; this may explain in our patient the head circumference $>3 \mathrm{SD}$ below the population mean, with a height lower than expected according to the height of his parents. However, the height seen in Seckel syndrome/primary dwarfism is usually lower (with intervals between 4 and 12 SDs below the population mean $)^{2}$.

Several genes are associated with autosomal recessive $\mathrm{MCPH}$. In the majority of cases, the brain structure is normal, and the most frequent anomaly is a small brain with small gyri $^{1-4,13}$. A simplified gyral pattern is frequent in patients with pathogenic variants in $A S P M^{3,4}$, WDR62 $2^{14}$ and $C I T^{3,4}$. Patients with WDR62 pathogenic variants show a wide spectrum of malformations, ranging from a small brain with a simplified gyral pattern to severe major brain structural malformations ${ }^{3,4,14}$. Cerebellar hypoplasia has been described in patients with CDK5RAP2, ASPM, CENPE, SASS6, MFSD2A, and CIT pathogenic variants ${ }^{2,3,15}$. Our patient has characteristics of typical MCPH: head circumference $>3$ SD below the population mean $(-5.42 \mathrm{SD})$, and cerebral neuroimaging showing bilateral migration disorder with heterotopia in the absence of other concomitant findings.

CENPJ is a 17-exon gene mapping at 13q12.2, which encodes centromere protein J, or CENPJ, a protein localized to centrosomes in interphase and to the spindle poles during mitosis ${ }^{3}$. Cho et al. observed that the depletion of CENPJ protein disrupts centrosome integrity and that cells lacking CENPJ arrest in mitosis with multipolar spindles ${ }^{16}$. The centrosome plays a key role in regulating cell division, functioning as a microtubule organizing center. Bond et al. proposed that a centrosomal mechanism is responsible for determining brain size $^{9}$. CENPJ interacts with proteins involved in $\mathrm{MCPH}$, such as CEP135, CEP152, WDR62, ASPM, and STIL, and binds microtubules ${ }^{17,18}$. In recent years, the function of 


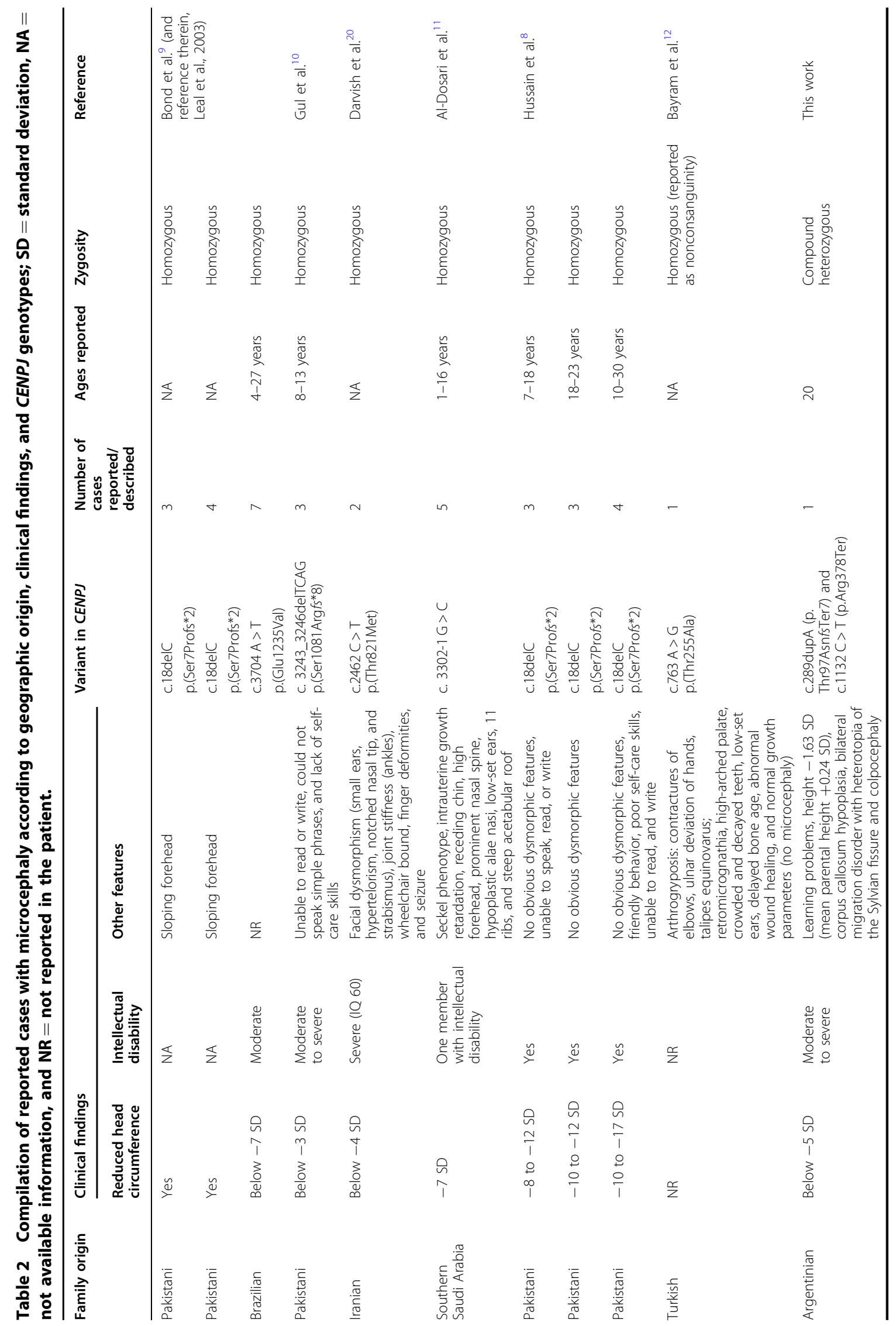


CENPJ in mice has been studied, confirming that this protein regulates progenitor division and neuronal migration in the cerebral cortex ${ }^{19}$.

In summary, our case represents a rather uncommon situation for the usual known context of $\mathrm{MCPH}$ and the CENPJ gene. These special characteristics include family origin (Argentinian), pedigree (nonconsanguineous), and genotype (a compound heterozygous case with two unreported variants predicting a truncated protein). NGS studies applied in a broader spectrum of clinical presentation of MCPH syndromes may discover additional patients and families with these characteristics and will help to provide accurate diagnosis, better phenotype delineation, and adequate genetic counseling.

\section{HGV database}

The relevant data from this Data Report are hosted at the Human Genome Variation database at https://doi.org/10.6084/m9.figshare.hgv.2858. https://doi. org/10.6084/m9.figshare.hgv.2861.

\section{Author details \\ 'Department of Clinical and Molecular Genetics, Vall d'Hebron Barcelona Hospital Campus, Barcelona, Spain. ${ }^{2}$ Medicine Genetics Group, Vall d’Hebron Research Institute (VHIR), Vall d'Hebron Barcelona Hospital Campus, \\ Autonomous University of Barcelona, Barcelona, Spain. ${ }^{3}$ European Reference Network Craniofacial Anomalies and ENT disorders (ERN CRANIO)(member) and ERN ITHACA (affiliated), Barcelona, Spain. ${ }^{4}$ Pediatric Endocrinology Unit, Vall d'Hebron Research Institute (VHIR), Vall d'Hebron Barcelona Hospital Campus, Autonomous University of Barcelona, Barcelona, Spain. ${ }^{5}$ Centro de Investigación Biomédica en Red de Enfermedades Raras (CIBERER), Instituto de Salud Carlos III, Barcelona, Spain. ${ }^{6}$ Neuromuscular and Mitochondrial Pathology Group, Vall d'Hebron Research Institute (VHIR), Vall d'Hebron Barcelona Hospital Campus, Autonomous University of Barcelona, Barcelona, Spain}

\section{Conflict of interest}

The authors declare that they have no conflict of interest.

\section{Publisher's note}

Springer Nature remains neutral with regard to jurisdictional claims in published maps and institutional affiliations.

Received: 17 December 2019 Revised: 20 April 2020 Accepted: 27 April 2020.

Published online: 8 June 2020

\section{References}

1. Abuelo, D. Microcephaly syndromes. Sem. Pediatr. Neurol. 14, 118-127 (2007).

2. Verloes A., et al. Primary Autosomal Recessive Microcephalies and Seckel Syndrome Spectrum Disorders (eds Adam M. P., Ardinger H. H., Pagon R. A., et al.) (GeneReviews ${ }^{\circledR}$ [Internet]. Seattle (WA): University of Washington, Seattle; 1993-2019).https:/www.ncbi.nlm.nih.gov/books/NBK9587/

3. Naveed, M. et al. Comprehensive review on the molecular genetics of autosomal recessive primary microcephaly (MCPH). Genet. Res. (Camb.) 100, e7 (2018).

4. Zaqout, S., Morris-Rosendahl, D. \& Kaindl, A. M. Autosomal recessive primary microcephaly (MCPH): an update. Neuropediatrics 48, 135-142 (2017).

5. OMIM (accessed August 2019); https://www.omim.org/search/? index $=$ entry\&search $=$ microcephaly+autosomal + recessive\&sort=score +desc\%2C+prefix_sort+desc\&start=1\&limit=10.

6. Shaheen, R. et al. Genomic and phenotypic delineation of congenital microcephaly. Genet. Med. 21, 545-552 (2019).

7. Richards, S. et al. Standards and guidelines for the interpretation of sequence variants: a joint consensus recommendation of the American College of Medical Genetics and Genomics and the Association for Molecular Pathology. Genet. Med. 17, 405-424 (2015).

8. Hussain, S., Bakhtiar, M. \& Farooq, M. et al. Genetic heterogeneity in Pakistani microcephaly families. Clin. Genet. 83, 446-451 (2013).

9. Bond, J. et al. A centrosomal mechanism involving CDK5RAP2 and CENPJ controls brain size. Nat. Genet. 37, 353-355 (2005).

10. Gul, A. et al. A novel deletion mutation in CENPJ gene in a Pakistani family with autosomal recessive primary microcephaly. J. Hum. Genet. 51, 760-764 (2006).

11. Al-Dosari, M. S., Shaheen, R., Colak, D. \& Alkuraya, F. S. Novel CENPJ mutation causes Seckel syndrome. J. Med. Genet 47, 411-414 (2010 Jun).

12. Bayram, Y. et al. Molecular etiology of arthrogryposis in multiple families of mostly Turkish origin. J. Clin. Invest. 126, 762-778 (2016).

13. Saheem, M. et al. Molecular genetics of human primary microcephaly: an ovenview. BMC Med. Genomics 8, S4 (2015).

14. Bilgüvar, K. et al. Whole-exome sequencing identifies recessive WDR62 mutations in severe brain malformations. Nature 467, 207-210 (2010).

15. Woods, C. G., Bond, J. \& Enard, W. Autosomal recessive primary microcephaly (MCPH): a review of clinical, molecular, and evolutionary findings. Am. J. Hum. Genet. 76, 717-728 (2005).

16. Cho, J. H., Chang, C. J., Chen, C. Y. \& Tang, T. K. Depletion of CPAP by RNAi disrupts centrosome integrity and induces multipolar spindles. Biochem. Biophys. Res. Commun. 339, 742-747 (2006).

17. Barbelanne M., Tsang W. Y. Molecular and cellular basis of autosomal recessive primary microcephaly. Biomed. Res. Int. 2014 (2014).

18. Jayaraman, D. et al. Microcephaly proteins Wdr62 and Aspm define a mother centriole complex regulating centriole biogenesis, apical complex, and cell fate. Neuron 92, 813-828 (2016).

19. Garcez, P. P., Diaz-Alonso, J., Crespo-Enriquez, I., Castro, D., Bell, D. \& Guillemot, F. Cenpj/CPAP regulates progenitor divisions and neuronal migration in the cerebral cortex downstream of Ascl1. Nat Commun. 6, 6474 (2015).

20. Darvish, H. et al. A clinical and molecular genetic study of 112 Iranian families with primary microcephaly. J. Med. Genet. 47, 823-828 (2010). 